\title{
Endoluminal transgastric endoscopic anastomosis of the gallbladder using an anchoring self-expand- ing metal stent
}

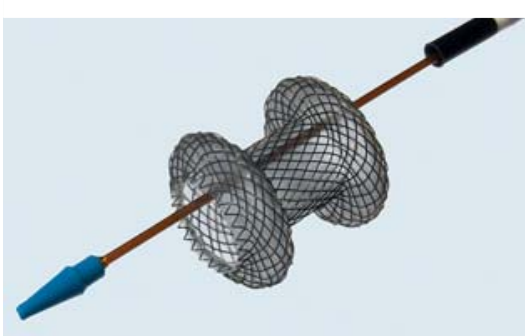

Fig. 1 The novel lumen-apposing self-expandable stent used to drain the gallbladder in an 86-year-old woman with acute cholecystitis.

An 86-year-old woman with a history of diabetes mellitus type 2, hypertension, and coronary artery disease was admitted for acute cholecystitis. On presentation she had abdominal pain and fever. Physical examination was remarkable for epigastric and right upper quadrant tenderness. Laboratory data showed leukocytosis (16000 cells/ $\mu \mathrm{L}$ with left shift; normal range $4500-10000$ cells $/ \mu \mathrm{L})$. An abdominal ultrasound confirmed acute cholecystitis because of gallstone disease. The patient was started on intravenous ceftriaxone and metronidazole and she refused surgery repeatedly. Due to the risks of gallbladder hydrops or rupture, we drained the gallbladder endoscopically using a self-expandable metal prosthesis with two flanged ends (Axios, Xlumena, Mountain View, California, USA) ( $\bullet$ Fig. 1). This novel lumen-apposing stent has been previously used to drain pseudocysts and has provided excellent results when used for draining nonadherent extraintestinal fluid collections [1]. The patient underwent the procedure after five days of antibiotic therapy.

Following conscious sedation with propofol, the endoscopic ultrasound (EUS)scope was advanced into the antrum and the gallbladder was located ( Fig. 2 ). The gallbladder wall was then punctured with a trocar (needle) under EUS guidance (๑ig.3a; Navix, Xlumena, Mountain View, California, USA). The position of the needle tip within the gallbladder was well visualized using EUS ( Fig. $\mathbf{3 b}$ ). The catheter of the Navix trocar was then advanced by turning the knob on its handle. A J-shaped 0.035-inch guide wire (Jagwire, Boston Scientific, Natick, Massachu-

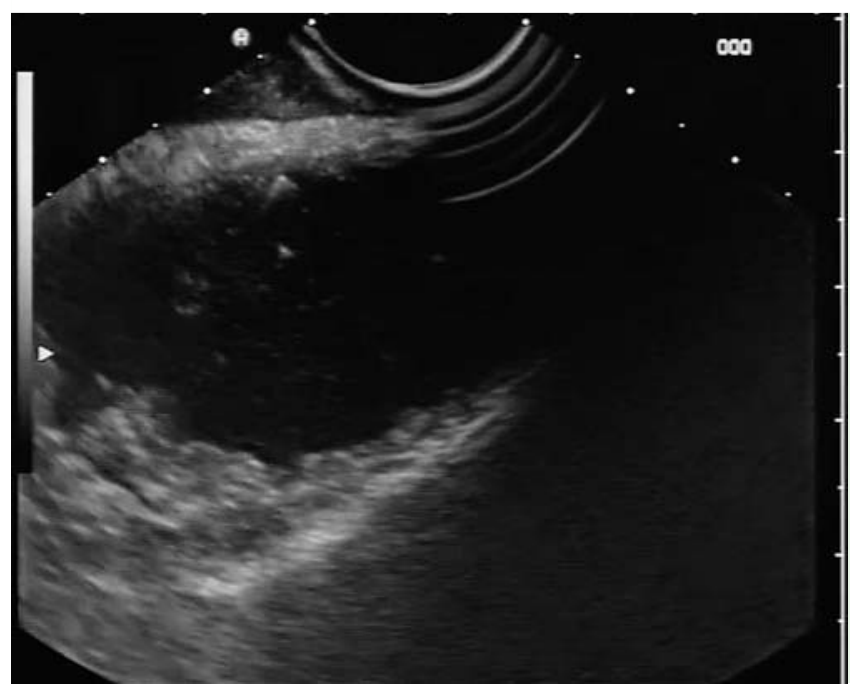

Fig. 2 Endoscopic ultrasound (EUS) view of the endoscope and gallbladder.

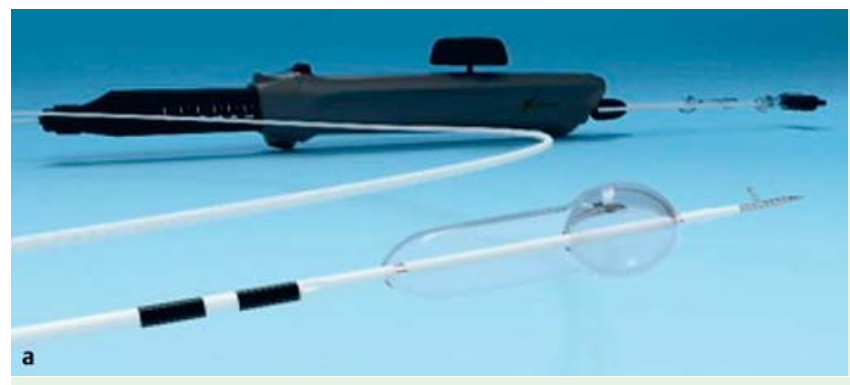

Fig. 3 a The Navix trocar.

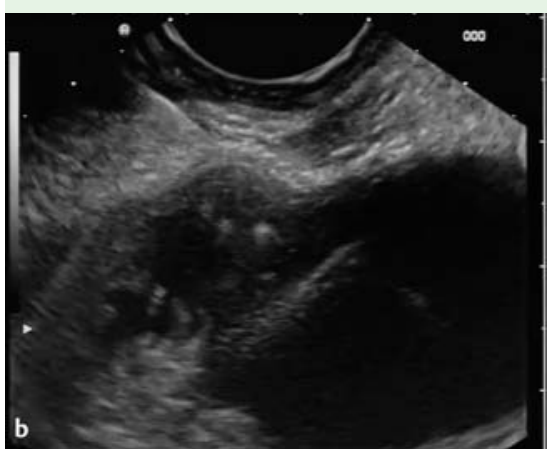

b Endoscopic ultrasound (EUS) view of the trocar within the gallbladder.

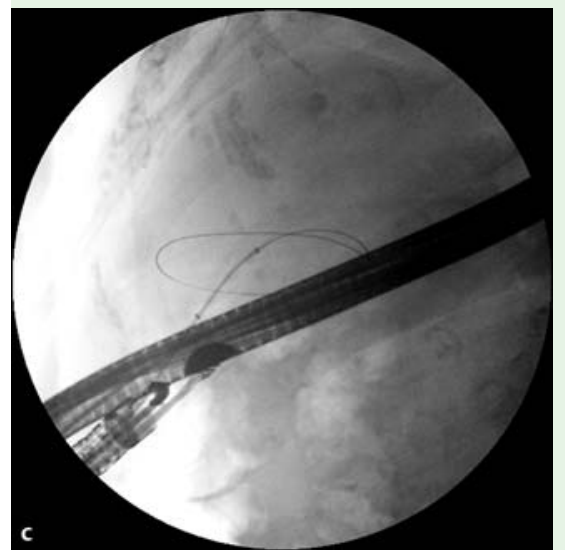

c Fluoroscopic view of the guide wire being advanced through the gallbladder. 

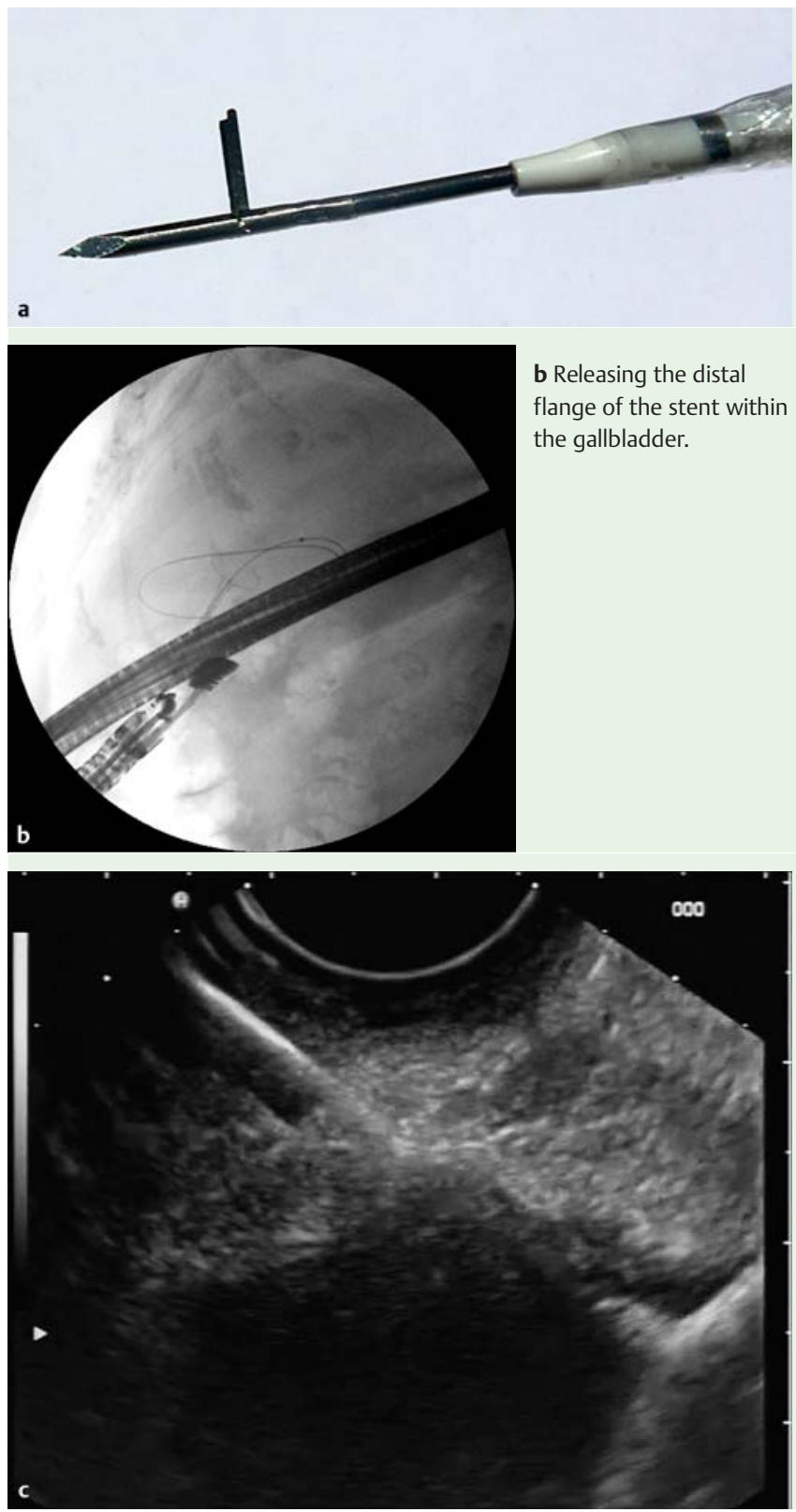

Fig. 4 a The Axios stent system.

c Endoscopic ultrasound (EUS) view clearly showing the deployed stent.

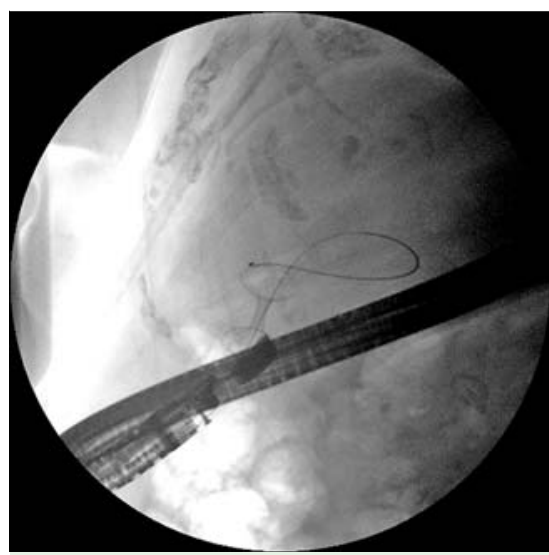

Fig. 5 Releasing the proximal flange of the stent in the stomach.

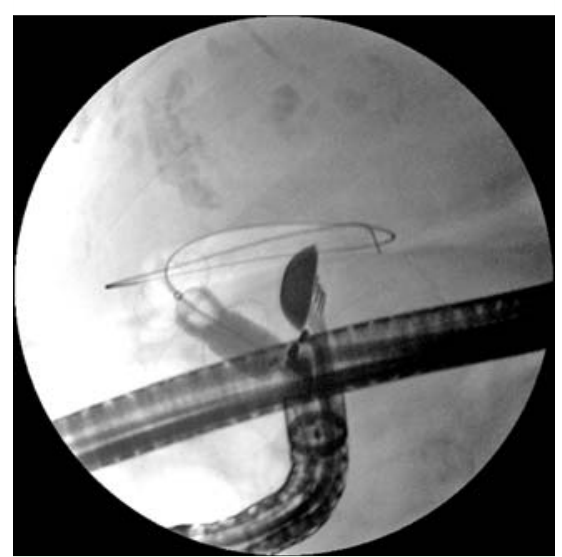

Fig. 6 Dilating the lumen-apposing stent. setts, USA) was then advanced through the Navix into the gallbladder and visualized using fluoroscopy ( Fig.3c). The Navix was then removed from the EUSscope and the Axios system ( $\bullet$ Fig.4a) advanced over the guide wire. The Axios catheter passed into the gallbladder without further dilation because of the 3.5- $\mathrm{mm}$ incision created by the Navix trocar and was advanced deeply into the gallbladder. The distal flange of the prosthesis was then released within the gallbladder ( Fig.4b). The Axios is highly echogenic and the deployment is easily observed with EUS ( Fig.4c). The Axios catheter was slowly repositioned (i.e., pulled back) for release of the proximal flange in the stomach, which is indicated by a black marker band on the catheter. Under both direct endoscopic and fluoroscopic visualization, the proximal flange was released ( $\bullet$ Fig. 5). The stent was dilated to $10 \mathrm{~mm}$ using the balloon of the delivery system ( Fig.6). Shortly afterwards, an ultraslim gastroscope (EVIS EXERA II video gastroscope GIF-XP-180N, $110 \mathrm{~cm}$ working length, $5.5 \mathrm{~mm}$ outer diameter, $2.0 \mathrm{~mm}$ working channel, Olympus, Hamburg, Germany) was passed through the newly placed lumen-apposing stent for direct cholecystoscopy, stone removal, and biopsy ( $\bullet$ Fig. 7a). The fluoroscopic view depicts the scope in retroflexion inside of the gallbladder ( Fig. 7 b). Following the procedure, the patient recovered well and was discharged 24 hours later.

Cholecystectomy is the treatment of choice for acute cholecystitis. Current options for treating patients who are poor surgical candidates or refuse surgery include percutaneous or surgical cholecystostomy [2]. However, there is a need for alternatives to cholecystectomy for these patients. Our present case is interesting for several reasons. First, we demonstrate the feasibility of utilizing endoscopy as a solution to a surgical problem. Percutaneous cholecystostomy is an option for draining an inflamed gallbladder [2]. However, accessing this organ through a natural orifice (i.e., mouth) and creating an internal anastomosis provides a more physiologic answer to the problem, as the bile will continue to flow into the gastrointestinal lumen, as shown in experimental studies [3]. Thus, this approach fully follows the principles of natural orifice translumenal endoscopic surgery (NOTES), i.e., finding an anatomic solution to a surgical problem with endoscopic methods using a natural orifice to access the gallbladder. 

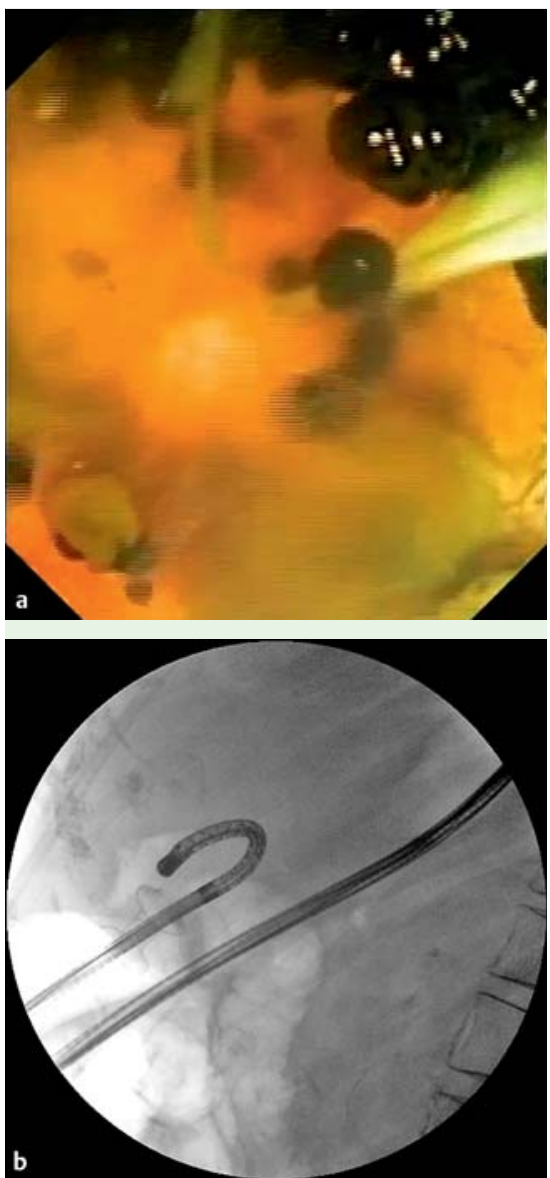

Fig.7 a A gastroscope being passed through the lumen-apposing stent for direct cholecystoscopy, stone removal, and biopsy. b Fluoroscopic view showing the scope in retroflexion inside the gallbladder.
We are not aware of any previous report documenting this technique.

Second, we confirm the concept of "anastomosis creation" using endoscopic methods. Although placement of a straight selfexpanding metal stent, or a straight or curved plastic stent, can lead to communication between two hollow cavities, the advantage of the novel lumen-apposing stent used in the present case is that both cavities remain held together by its double-flanged ends. That is, the flanges, or "tulips," on each end of the prosthesis produce a bilateral contracting force that impeded the separation of both cavity walls, thus imparting lumen-to-lumen anchorage [1].

Third, we show that due to the excellent deployment and expansion of the stent and its capacity to keep the stomach and gallbladder together, it can be used immediately as a channel to enter the gallbladder lumen with an endoscope. Thus, during direct cholangioscopy we were able to aspirate pus and bile and remove stones.

In summary, we believe that transgastric flexible endoscopic surgery might offer advantages over open and laparoscopic surgery or percutaneous cholecystostomy in patients with acute cholecystitis at high risk of surgical complications. We also believe that the creation of internal anastomosis in nonadherent or partially adherent lumens (i.e., "anastomotic connectivity") using advanced prosthesis such as the present double-flanged stent, in addition to become a life-saving procedure, may be a more physiologic solution for the problem of gallbladder drainage.

Endoscopy_UCTN_Code_TTT_1AS_2AG

Competing interests: None

\section{K. Mönkemüller ${ }^{1,2}$, M. Zabielski ${ }^{1}$, A. Didowacz-Grollmann ${ }^{1}$, C. von Gru- challa', H. Neumann ${ }^{3}$, K. Vormbrock ${ }^{1}$}

${ }^{1}$ Department of Internal Medicine, Gastroenterology, and Infectious

Diseases, Marienhospital Bottrop, Germany

2 Division of Gastroenterology, Hepatology, and Infectious Diseases, Otto-von-Guericke University, Magdeburg, Germany

${ }^{3}$ Department of Medicine I, University of Erlangen-Nuremberg, Erlangen, Germany

\section{References}

1 Binmoeller KF, Shah J. A novel lumen-apposing stent for transluminal drainage of nonadherent extraintestinal fluid collections. Endoscopy 2011; 43: 337-342

2 Joseph T, Unver K, Hwang GL et al. Percutaneous cholecystostomy for acute cholecystitis: ten-year experience. J Vasc Interv Radiol 2012; 23 (Suppl. 01): 83-88 e1

3 Park PO, Bergström M, Ikeda $K$ et al. Experimental studies of transgastric gallbladder surgery: cholecystectomy and cholecystogastric anastomosis (videos). Gastrointest Endosc 2005; 61: 601-606

Bibliography

DOI http://dx.doi.org/

10.1055/s-0032-1325776

Endoscopy 2013; 45: E164-E166

(c) Georg Thieme Verlag KG

Stuttgart · New York

ISSN 0013-726X

\section{Corresponding author}

K. Mönkemüller

Department of Internal Medicine,

Gastroenterology, and Infectious Diseases

Marienhospital Bottrop

Josef-Albers-Str. 70

46236 Bottrop

Germany

Fax: +49-204-11061019

moenkemueller@yahoo.com 